\title{
Scaling behaviour in temporal fluctuations of crustal seismicity in Taiwan
}

\author{
L. Telesca ${ }^{1}$, C.-C. Chen ${ }^{2}$, and Y.-T. Lee ${ }^{2}$ \\ ${ }^{1}$ Institute of Methodologies for Environmental Analysis, National Research Council, C. da S. Loja, 85050 Tito (PZ), Italy \\ ${ }^{2}$ Graduate Institute of Geophysics, National Central University, Jhongli 320, Taiwan
}

Received: 8 October 2009 - Accepted: 4 November 2009 - Published: 10 December 2009

\begin{abstract}
The crustal seismicity of Taiwan was investigated by means of the Allan Factor analysis and Count-based Periodogram, which allow to identify scaling behaviour in point processes and to quantify their temporal fluctuations by means of the estimate of the scaling exponent. Our findings point out to the presence of two time-scaling regions in the crustal Taiwanese seismicity. The first region, involving the intermediate timescales can be probably linked with aftershock activity, while the second region, involving the large timescales could be related with the background seismicity.
\end{abstract}

\section{Introduction}

It is known from many laboratory observations that earthquake occurrence is associated with a high level of stress on the fault. The spatiotemporal pattern in seismicity reflects a space-time correlation associated with the crustal stress and strain fields. Ideas relating to anomalous changes in seismicity rates prior to a major earthquake thus have been frequently proposed and widely discussed in the literature (e.g. Chen et al., 2005). Consequently, understanding of spatial and temporal behaviors of seismicity is fundamental of earthquake prediction.

Earthquakes are a typical example of complex system, where spatial, temporal and energy parameters are powerlaw distributed. The existence of power-laws qualifies earthquakes as a good example of fractal systems. The investigation of the time-fractal properties of earthquakes, in order to gain insight into the dynamics of seismicity, was performed using statistics like the Variance-Time curve (Telesca et al., 2000), Allan Factor (Telesca et al., 2000), the Fano Factor (Telesca et al., 2003), the Detrended Fluctuation Analysis

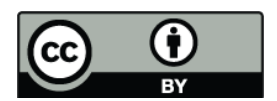

Correspondence to: C.-C. Chen (chencc@ncu.edu.tw)
(DFA) (Telesca and Lapenna, 2006). All these measures lead to the determination of a scaling exponent, estimated by a linear fitting procedure performed on the power-law statistics plotted in log-log scales. Such scaling exponent informs about the dynamical properties of an earthquake sequence, in terms of its correlation structures and its memory phenomena. Furthermore, the numerical value of the scaling exponent allows to determine quantitatively the strength of the temporal fluctuations of seismicity. Several studies regarded the discrimination between Poissonian and clusterized sequences (Telesca et al., 2001), spatial variability of time-clustering behaviour (Telesca et al., 2003), aftershockrelated patterns (Telesca et al., 2004), depth-dependent variability related with the brittle and ductile behaviour of the crust versus depth and magnitude-variability of the property of time-clusterization (Telesca et al., 2008). Therefore, the analysis of the time-scaling properties of earthquakes could characterize the main features of seismicity and to bring us insight the inner dynamics of seismotectonic activity.

The objective of this paper is to analyze the time-scaling properties of the crustal seismicity of Taiwan by means of second-order fractal tools, in order to better describe its dynamics.

\section{Seismo-tectonic settings of Taiwan}

The Island of Taiwan is located on the active boundary between the Philippine Sea plate and the Eurasian plate along the western Circum-Pacific seismic belt (Fig. 1). The overall plate configuration in the vicinity of Taiwan is well defined by the prevalent seismic activity in the region. The Eurasian plate is subducting eastward beneath the Philippine Sea plate along the Manila Trench to the south of Taiwan, whereas the Philippine Sea plate is subducting northwestward under the Eurasian plate along the Ryukyu Trench to the northeast of Taiwan. In the vicinity of Taiwan, the relative plate

Published by Copernicus Publications on behalf of the European Geosciences Union. 


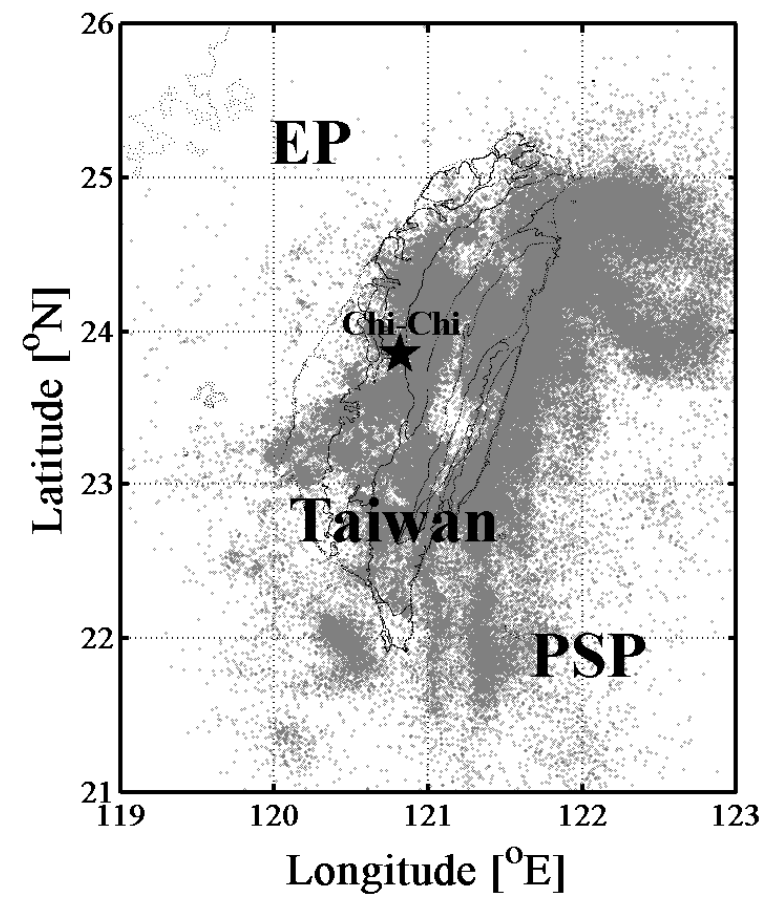

Fig. 1. Epicentral distribution of the selected seismic events from the Taiwanese CWB catalogue. $\mathrm{EP}=$ Euriasian plate; PSP $=$ Philippine Sea plate. Star indicates the epicenter of the 1999 M7.6 Chi-Chi earthquake.

convergence velocity between the Philippine Sea plate and Eurasian plate is about $82 \mathrm{~km}$ per million years and approximately in the direction $\mathrm{N} 50^{\circ} \mathrm{W}$ (Yu et al., 1997). Tectonically, most of the Taiwan region is under the NW-SE compression. The rapid plate collision is responsible for the complex geological setting and the rugged topography.

The collision of those two plates also causes the Taiwan area to be a high seismicity area and many disastrous earthquakes had occurred in this area in the past. For example, the Chi-Chi earthquake with moment magnitude $\left(M_{w}\right) 7.6$ struck central Taiwan on 21 September 1999 (or at 17:47 Coordinated Universal Time, 20 September 20) which was the largest earthquake that occurred on the Taiwan Island in the 20th century (Chang et al., 2000; Shin, 2000; Teng et al., 2001). The earthquake initiated at $\left(23.853^{\circ} \mathrm{N}\right.$, $120.816^{\circ} \mathrm{E}$ ), near the small town of Chi-Chi, with a focal depth of $8 \mathrm{~km}$. This event struck the central Taiwan heavily and resulted in serious damage (Tsai et al., 2001). Seismic waves generated by the Chi-Chi rupturing also inflicted severe damage in the Taipei basin, which is as far as $150 \mathrm{~km}$ away from the epicenter. As of 13 October 1999 the officially released casualty figures were 2333 dead and 10002 injured. At the end of 1999 the Ministry of Interior of Taiwan reported that the death toll was 2412 and the number of injury stood at 11305 . Thousands of houses collapsed and more than 100000 people were left homeless.
Based on the tectonics and seismic characteristics, the Taiwan region can be classified into four different seismogenic zones (Wu and Chen, 2007): (A) Western Seismic Zone: located within the Eurasia Plate. Most of the earthquakes in this zone can be associated with active faults; (B) Southwestern Seismic Zone: located mainly in the South China Sea block of the Eurasian Plate. In terms of seismic activity, this is the least active zone among the four; (C) Northeastern Seismic Zone: associated with the Ryukyu subduction system and shows high seismicity with occasional damaging earthquakes; and (D) Southeastern Seismic Zone: mainly caused by the collision of the Eurasia Plate and the Luzon island arc on the Philippine Sea Plate. Most of the seismic activity in the Taiwan region, including many large earthquakes, occurs in this area. For details the readers are referred to the paper of $\mathrm{Wu}$ and Chen (2007) and references therein.

\section{The methods}

A seismic sequence can be considered as a realization of a stochastic point process. A stochastic point process describes events that occur at some random locations in time (Cox and Isham, 1980) and is completely defined by the set of the event times. A point process may be called fractal when a number of its relevant statistics exhibit scaling with related scaling exponents, that indicate the represented phenomenon contain clusters of points over a relatively large set of timescales (Teich et al., 1996). The standard method to investigate the presence of clustering in a time series is the power spectral density (PSD), which informs on how the power of the process is concentrated at various frequency bands. The PSD is calculated by means of the Fourier transform. If the process has scaling properties the PSD follows a power-law behaviour $S(f) \propto f^{-\alpha}$, with the so-called fractal exponent $\alpha$ measuring the strength of the clustering. If the point process is Poissonian, the event occurrence times are uncorrelated; for this memoryless process $\alpha \approx 0$. On the other side, $\alpha \neq 0$ is typical of point processes with self-similar behaviour; selfsimilar meaning that parts of the whole can be made to fit to the whole in some way by scaling (Mandelbrot, 1983). Thus, we can understand that the estimation of the fractal exponent $\alpha$ for a seismic sequence plays an important role in the general dynamical characterisation of the mechanism underlying seismicity.

The analysis of the temporal properties of an earthquake sequence implies that this sequence is appropriately described, in order to apply to it statistical methods able to reveal the presence of clusterisation. A discrete-time process can be derived from the stochastic point process forming its relative counting process, where the time axis is divided into equally spaced contiguous counting windows of duration $\tau$ to produce a sequence of counts $\left\{N_{k}(\tau)\right\}$, where $N_{k}(\tau)$ represents the number of events falling into the $k$-th window of 
duration $\tau$. The duration $\tau$ of the window is called counting time or timescale. This approach considers the fire events as the events of interest and assumes that there is an objective clock for the timing of the events.

A measure, related to the variability of successive counts, useful to detect the event clustering in a point process, is the Allan Factor (AF) (Allan, 1966), defined as the variance of successive counts for a specified counting time $\tau$ divided by twice the mean number of events in that counting time

$\operatorname{AF}(\tau)=\frac{<\left(N_{k+1}(\tau)-N_{k}(\tau)\right)^{2}>}{2<N_{k}(\tau)>}$.

This measure reduces the effect of possible nonstationarity of the point process, because it is defined in terms of the difference of successive counts. Varying the timescale $\tau$ allows producing a relationship between $\operatorname{AF}(\tau)$ and $\tau$, useful to detect scaling behavior in the sequence.

The AF of a fractal point process varies with the counting time $\tau$ with a power-law form:

$\operatorname{AF}(\tau)=1+\left(\frac{\tau}{\tau_{1}}\right)^{\alpha}$

with $0<\alpha<3$ over a relatively large range of counting times $\tau$ (Thurner et al., 1997); $\tau_{1}$ is the fractal onset time for the $\mathrm{AF}$ and is estimated as the crossover timescale between Poissonian and scaling behaviors. AF assumes values near unity for Poisson processes.

The count-based Periodogram (PG) (Lowen and Teich, 1996), that is the periodogram of the sequence of the counts, is another statistical measure that allows estimating the fractal exponent $\alpha$. This measure performs an estimate of the power spectral density, which gives information on how the power of the process is concentrated at various frequency bands. The calculation of the PG by means of a count-based approach implies the division of the total observation period into a number $N$ of nonoverlapping windows of length $T_{W}$. The sequence of the counts is then Fourier transformed and the PG is obtained by the squares of the coefficients of the Fourier representation of the series of counts. For point processes with scaling properties the PG decreases as a powerlaw function of the frequency $f$ over a significant range of frequencies, $S(f) \propto f^{-\alpha}$. Of course, for a finite size fractal real process the power spectral density behaves as an inverse power-law function in a limited range of frequencies, approaching an asymptotic value at high frequencies, at which the behaviour of the process can be considered Poissonian. The numerical value of $\alpha$ is an indicator of the presence of clusterization in the process (Thurner et al., 1997). If the point process is Poissonian, the occurrence times are uncorrelated; for this memoryless process, the PG is approximately flat for any frequency bands and $\alpha \approx 0$. On the other side, $\alpha \neq 0$ is typical of point processes with scaling behavior. This method introduces a bias at higher frequencies, since the fine time resolution information is lost as a result of the

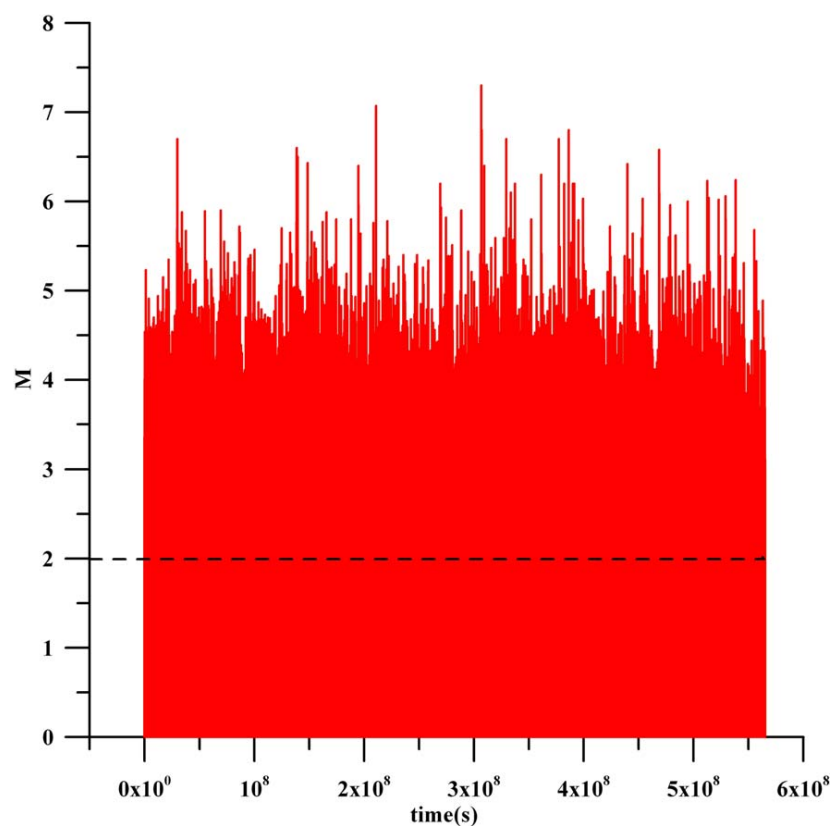

Fig. 2. Magnitude-time plot of the selected seismic events from the Taiwanese CWB catalogue. We have shifted the occurrence time of the first event as zero seconds.

minimum count-window size. But, the estimation of the exponent $\alpha$ principally involves lower frequencies where this bias is negligible.

Lowen and Teich (1996) showed the correspondence between the two scaling exponents, estimated by the $\mathrm{AF}$ and the PG methods, in the range $0<\alpha<3$, being identical for an ideal fractal stochastic point process. The two methods convey the same information, regarding the presence of correlated structures in the temporal fluctuations of point processes, but in different domains, time for the AF and frequency for the PG. In some cases the use of PG is necessary for $\alpha \geq 3$, that cannot be estimated by the AF method due to its saturation effect (Lowen and Teich, 1996).

\section{Results and discussion}

The seismic data, covering the period from 1990 to 2007, were extracted from the earthquake catalogue maintained by the Central Weather Bureau (CWB) in Taiwan, and regard all Taiwan. The epicenters of the earthquakes whose depth is less than $30 \mathrm{~km}$ are shown in Fig. 1. Figure 2 shows the temporal distribution in magnitude of Taiwanese crustal seismicity. The black horizontal line indicates the completeness magnitude, as estimated by the Gutenberg-Richter analysis (Fig. 3).

The results of the AF analysis are shown in Fig. 4. The AF curve increases with the counting time $\tau$, indicating the presence of correlated temporal fluctuations. In particular several timescale regions can be approximately identified. 


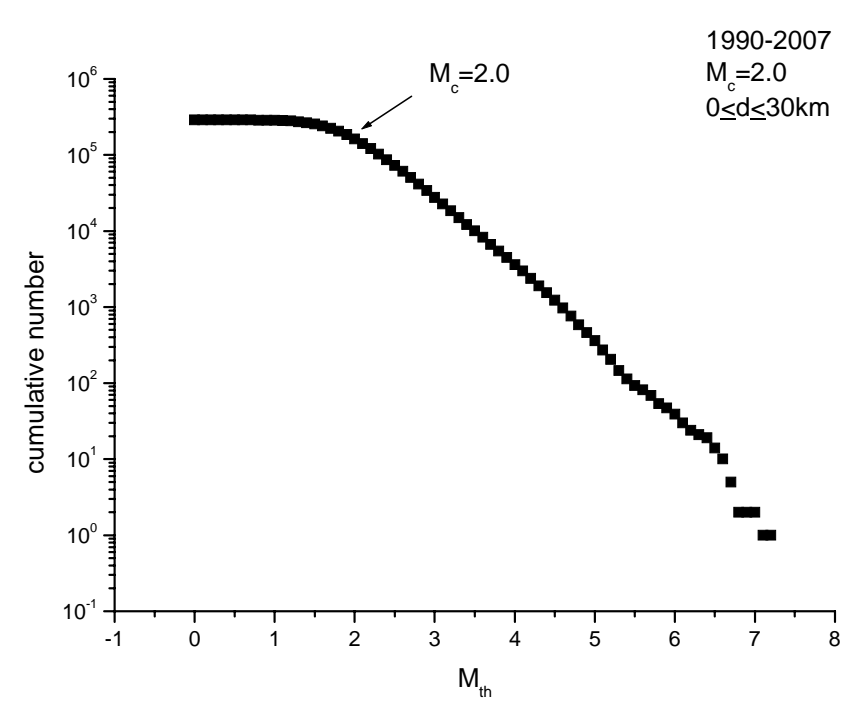

Fig. 3. Gutenberg-Richter analysis performed on the Taiwanese catalogue. The selected seismic events span from 1990 to 2007 with a depth between 0 and $30 \mathrm{~km}$. The estimated completeness magnitude is 2.0 .

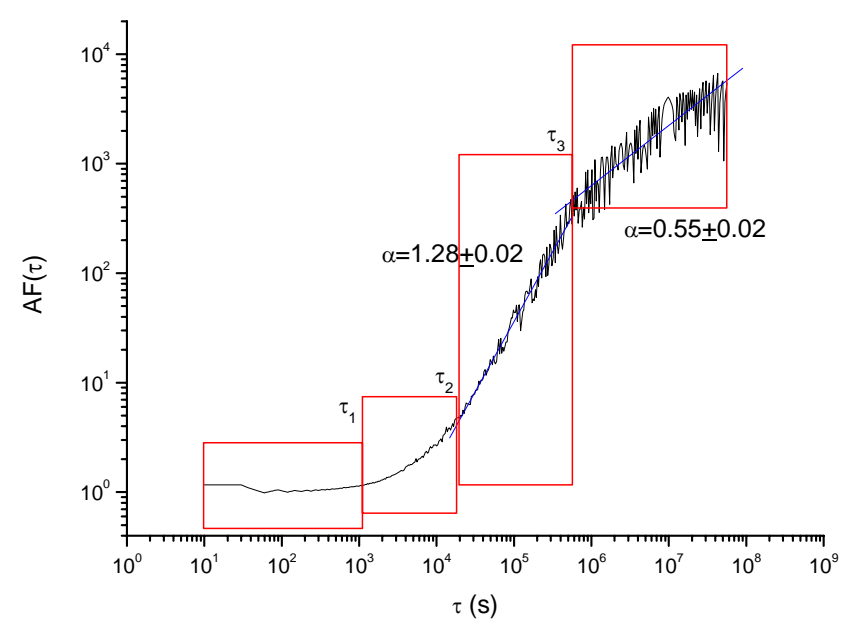

Fig. 4. AF results for the Taiwanese seismicity. The AF curve is characterized by four time regions: a) Possonian (up to $\tau_{1} \sim 15 \mathrm{~min}$ ); b) transition (from $\tau_{1}$ to $\tau_{2} \sim 30 \mathrm{~min}$ ); c) scaling (from $\tau_{2}$ to $\tau_{3} \sim 6-$ 7 days), with $\alpha \sim 1.3$ and d) scaling ( $\tau>\tau_{3}$ ), with $\alpha \sim 0.6$.

i) The first region involves the low timescales up to $\tau_{1} \sim 15 \mathrm{~min}$; in this region the AF assumes approximately constant unity value, denoting Poissonian temporal fluctuations involving these timescales; the timescale $\tau_{1}$ can be interpreted as the fractal onset time, above which fractal behaviour can be detected. ii) The region between $\tau_{1}$ and $\tau_{2} \sim 30 \mathrm{~min}$ is a transition region between the Poissonian regime and the scaling regime, which ranges between $\tau_{2}$ and $\tau_{3} \sim 6-7$ days; the curvature in the transition region is due to the mutual effect of the approximately flat Poissonian behaviour of the first region and the approximately linear be-

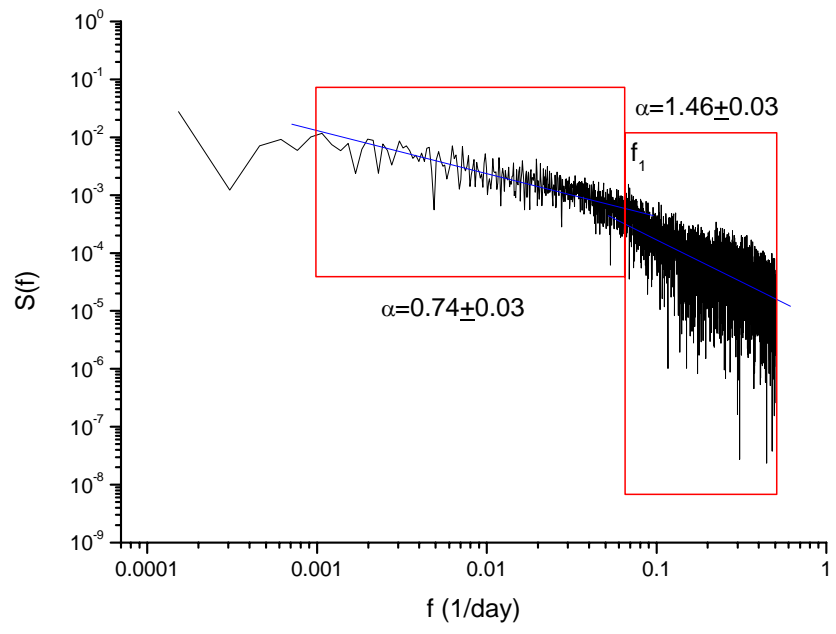

Fig. 5. PG results for the daily counts of the Taiwanese seismicity. The PG curve shows approximately two scaling regions with a crossover $\left(\tau_{c}=1 / f_{c}\right)$ at about $14-15$ days. The low frequency part of the PG has $\alpha \sim 0.7$, while the high frequency part of PG is characterized by $\alpha \sim 1.5$.

haviour shown by the successive scaling region. iii) The scaling region between $\tau_{2}$ and $\tau_{3}$; the value of the scaling exponent, estimated as the slope of the line fitting by the least square method the curve plotted in log-log scales, $\alpha \sim 1.28$, indicates a high degree of clusterization of the events. iv) The scaling region for timescales larger than $\tau_{3}$ has a lower value for the scaling exponent $\alpha$, approximately 0.55 , which, anyway, indicates a rather high degree of clusterization of the earthquakes.

The results obtained by applying the count-based periodogram (PG) are shown in Fig. 5. The PG was calculated by applying the Fourier Transform to the series of daily counts. The PG, plotted in log-log scales, decreases almost linearly respect to the frequency, revealing two scaling regions, separated by the frequency crossover of about 14-15 days. The $\alpha$-estimates performed by the PG are slightly different from those obtained by means of AF, probably due to bias that the PG method suffers. However, the estimates are consistent among them, and also the crossovers obtained by means the $\mathrm{AF}$ and the PG methods are in agreement.

The obtained results point out to a correlated behaviour in the Taiwanese seismicity. The presence of two different scaling regions, involving intermediate timescales and large timescales with different values for the scaling exponents, indicates the presence of at least two different mechanisms underlying the dynamics of the same seismic process. Very probably the first scaling range, up to 6-7 days or 14-15 days (depending on the AF or PG results, respectively), could describe aftershock temporal fluctuations, while the scaling range, involving the larger timescales, could characterize the temporal fluctuations of the background seismicity of Taiwan. Similar results were obtained by Telesca et al. (2002), 
where the Umbria-Marche (central Italy) seismicity, struck by the M5.8 earthquake in 1997, was analysed. Also in this case, two different scaling regions were identified, with quite similar values for the scaling exponents and crossover timescales.

\section{Conclusions}

The main results regarding the Taiwanese seismicity can be summarized as follows: (i) it is not Poissonian, and thus uncorrelated, because the AF and the PG are not approximately flat for any timescale; (ii) it has a twofold scaling temporal behaviour, with different values for the scaling exponent; (iii) the higher clusterization degree involves the intermediate timescales up to a timescale of the order of days, which is probably linked with aftershock mechanisms; (iv) similar dynamical behaviour with that shown by the seismicity of a different seismotectonic setting (central Italy) was observed.

Acknowledgements. L. T. acknowledges CNR and NSC for their support through the bilateral agreement for scientific cooperation. C. C. C. is grateful for research support from both the National Science Council (ROC) and the Institute of Geophysics (NCU, ROC).

Edited by: M. E. Contadakis

Reviewed by: M. Lovallo and A. Ramírez-Rojas

\section{References}

Allan, D. W.: Statistics of atomic frequency standards, Proc. IEEE, 54, 221-230, 1966.

Chang, C. H., Wu, Y. M., Shin, T. C., and Wang, C. Y.: Relocating the 1999 Chi-Chi Earthquake, Taiwan, Terr. Atmos. Ocean. Sci., 11, 581-590, 2000.

Chen, C. C., Rundle, J. B., Holliday, J. R., Nanjo, K. Z., Turcotte, D. L., Li, S. C., and Tiampo, K. F.: The 1999 ChiChi, Taiwan, earthquake as a typical example of seismic activation and quiescence, Geophys. Res. Lett., 32(22), L22315, doi:10.1029/2005GL023991, 2005.

Cox, D. R. and Isham, V.: Point Processes, Chapman and Hall, London, 1980.

Lowen, S. B. and Teich, M. C.: The Periodogram and Allan Variance reveal fractal exponents greater than unity in Auditorynerve Spike Trains, J. Acoust. Soc. Am., 99, 3585-3591, 1996.

Mandelbrot, B. B.: The Fractal Geometry of Nature, W. H. Freeman, New York, 1983.

Shin, T. C.: Some seismological aspects of the 1999 Chi-Chi earthquake in Taiwan, Terr. Atmos. Ocean. Sci., 11, 555-566, 2000.
Teich, M. C., Heneghan, C., Lowen, S. B., and Turcott, R. G.: Estimating the fractal exponent of point processes in bilogical systems using wavelet- and Fourier-transform methods, in: Wavelets in Medicine and Biology, edited by: Aldroubi, A. and Unser, M. CRC Press, Boca Raton, FL, 383-412, 1996

Telesca, L., Lapenna, V., Lovallo, M., and Macchiato, M.: Longrange time-correlation properties of seismic sequences, Chaos, Solitons \& Fractals, 21, 387-393, 2004.

Telesca, L., Cuomo, V., Lapenna, V., and Macchiato, M.: Analysis of the time-scaling behaviour in the sequence of the aftershocks of the Bovec (Slovenia) April 12, 1998 earthquake, Phys. Earth Planet. In., 120, 315-326, 2000.

Telesca, L., Cuomo, V., Lapenna, V., and Macchiato, M.: Statistical analysis of fractal properties of point processes modelling seismic sequences, Phys. Earth Planet. In., 125, 65-83, 2001.

Telesca, L., Cuomo, V., Lapenna, V., and Macchiato, M.: 1/f ${ }^{\alpha}$ fluctuations of seismic sequences, Fluctuations and Noise Letters, 2, L357-L367, 2002.

Telesca, L., Cuomo, V., Lapenna, V., and Vallianatos, F.: Selfsimilarity properties of seismicity in the Southern Aegean area, Tectonophysics, 32, 179-188, 2000.

Telesca, L. and Lapenna, V.: Measuring multifractality in seismic sequences, Tectonophysics, 423, 115-123, 2006.

Telesca, L., Lapenna, V., and Macchiato, M.: Investigatine the time-clustering properties in seismicity of Umbria-Marche region (central Italy), Chaos, Solitons \& Fractals, 18, 203-217, 2003.

Telesca, L., Lapenna, V., and Macchiato, M.: Spatial variability of the time-correlated behaviour in the Italian seismicity, Earth Planet. Sc. Lett., 212, 279-290, 2003.

Telesca, L., Lovallo, M., Lapenna, V., and Macchiato, M.: Spacemagnitude dependent scaling behaviour in seismic interevent series revealed by detrended fluctuation analysis, Physica A, 387, 3655-3659, 2008.

Teng, T. L., Tsai, Y. B., and Lee, W. H. K.: Preface to the 1999 ChiChi, Taiwan, earthquake dedicated issue, B. Seismol. Soc. Am., 91, 893-894, 2001.

Thurner, S., Lowen, S. B., Feurstein, M. C., Heneghan, C., Feichtinger, H. G., and Teich, M. C.: Analysis, Synthesis, and Estimation of Fractal-Rate Stochastic Point Processes, Fractals, 5, 565-596, 1997.

Tsai, Y. B., Yu, T. M., Chao, H. L., and Lee, C.: Spatial distribution and age dependence of human-fatality rates from the ChiChi, Taiwan, Earthquake of 21 September 1999, B. Seismol. Soc. Am., 91, 1298-1309, 2001.

Wu, Y. M. and Chen, C. C.: Seismicity patterns before and after the 1999 Chi-Chi, Taiwan, Mw 7.6 earthquake, Tectonophysics, 429, 125-132, 2007.

Yu, S. B., Chen, H. Y., and Kuo, L. C.: Velocity field of GPS stations in the Taiwan area, Tectonophysics, 274, 41-60, 1997. 